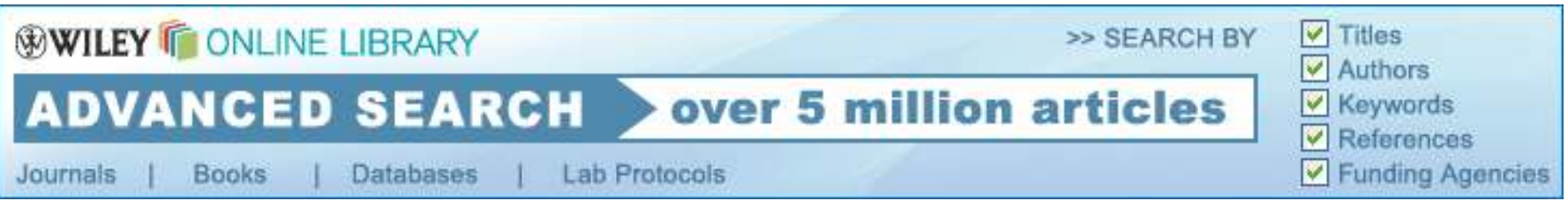

\title{
Phaeomoniella chlamydospora-induced Oxidative Burst in Vitis vinifera Cell Suspensions: Role of NADPH Oxidase and $\mathrm{Ca}^{2+}$
}

1. Marta R. M. Lima,

2. Alberto C. P. Dias

Article first published online: 22 DEC 2011

DOI: $10.1111 /$ j.1439-0434.2011.01871.x

(C) 2011 Blackwell Verlag GmbH

Issue

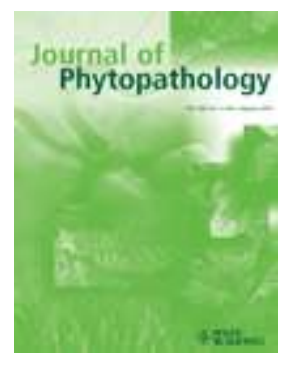

\section{Journal of Phytopathology}

Early View (Online Version of Record published before inclusion in an issue)(/journal/10.1111 (ISSN)1439-0434/earlyview)

Additional Information

\section{How to Cite}

Lima, M. R. M. and Dias, A. C. P. (2011), Phaeomoniella chlamydospora-induced Oxidative Burst in Vitis vinifera Cell Suspensions: Role of NADPH Oxidase and $\mathrm{Ca}^{2+}$. Journal of Phytopathology. doi: 10.1111/j.1439-0434.2011.01871.x

\section{Author Information}

Authors' address: Departamento de Biologia, CITAB - Centro de Investigação e Tecnologias Agro-Ambientais e Biológicas, Universidade do Minho, Campus de Gualtar, 4710-057 Braga, Portugal (correspondence to A. C. P. Dias. E-mail: acpdias@bio.uminho.pt(mailto:acpdias@bio.uminho.pt)) 


\section{Publication History}

1. Article first published online: 22 DEC 2011

2. Received April 21, 2011; accepted August 17, 2011

- Abstract

- Article(full)

- References(references)

- $\underline{\text { Cited By(citedby) }}$

View Full Article (HTML)(/doi/10.1111/j.1439-0434.2011.01871.x/full) Get PDF (283K)(/doi/10.1111 /j.1439-0434.2011.01871.x/pdf)

\section{Keywords:}

esca disease; measles; reactive oxygen species; grapevine; in vitro cultures; $\mathrm{Ca}^{2+}$ chelators; $\mathrm{Ca}^{2+} \mathrm{c}$

\section{Abstract}

The biphasic oxidative burst induced by Phaeomoniella chlamydospora extract (Pce) in Vitis vinifera (Vv) cell suspensions was investigated. Treatment of cell suspensions with diphenyleneiodonium chloride, an inhibitor of NADPH oxidase, prevented the Pce-induced biphasic reactive oxygen species (ROS) accumulation, suggesting that NADPH oxidase is the primary ROS source in the oxidative burst induced by Pce elicitation of $\mathrm{Vv}$ cells. The role of $\mathrm{Ca}^{2+}$ in the oxidative burst was also investigated using a $\mathrm{Ca}^{2+}$ chelator and several $\mathrm{Ca}^{2+}$ channel blockers. The treatment of $\mathrm{Vv}$ cell suspensions with the $\mathrm{Ca}^{2+}$ chelator ethylene glycol-bis(2-aminoethylether)-N, N, N'; N'-tetraacetic acid (EGTA) completely inhibited Pce-induced ROS accumulation, suggesting that $\mathrm{Ca}^{2+}$ availability is necessary for occurrence of the induced oxidative burst. However, only the $\mathrm{Ca}^{2+}$ channel blocker ruthenium red strongly inhibited the Pce-induced ROS accumulation, suggesting that the specific $\mathrm{Ca}^{2+}$ channel types from which $\mathrm{Ca}^{2+}$ influx is originated also play an important role in the Pce-induced oxidative burst. Furthermore, $\mathrm{Ca}^{2+}$ availability seems to be necessary for the Pce-induced activity of NADPH oxidase.

View Full Article (HTML)(/doi/10.1111/j.1439-0434.2011.01871.x/full) Get PDF (283K)(/doi/10.1111 j.1439-0434.2011.01871.x/pdf)

\section{More content like this}

Find more content:

- $\underline{\text { like this article(/advanced/search/results?articleDoi=10.1111/j.1439-0434.2011.01871.x\& }}$ $\underline{\text { scope }=\text { allContent } \& \text { start }=1 \& \text { resultsPerPage }=20)}$

Find more content written by:

- Marta R. M. Lima(/advanced/search/results?searchRowCriteria[0].queryString="Marta R. M. Lima"\&searchRowCriteria[0].fieldName=author\&start=1\&resultsPerPage $=20$ )

- Alberto C. P. Dias//advanced/search/results?searchRowCriteria[0].queryString="Alberto C. P. Dias"\&searchRowCriteria[0].fieldName $=$ author\&start=1\&resultsPerPage $=20$ )

- All Authors//advanced/search/results?searchRowCriteria[0].queryString="Marta R. M. Lima" "Alberto C. P. Dias"\&searchRowCriteria[0].fieldName=author\&start=1\&resultsPerPage=20) 